\title{
Talking About the Prevention and Control and Precautions of African Hog in China
}

\author{
Shi Haidong \\ Agricultural Service Center of Haiyong Town People's Government, Haimen, China \\ Email address: \\ js_zsl@163.com \\ To cite this article: \\ Shi Haidong. Talking About the Prevention and Control and Precautions of African Hog in China. Biomedical Sciences. \\ Vol. 5, No. 2, 2019, pp. 14-19. doi: 10.11648/j.bs.20190502.11
}

Received: July 31, 2019; Accepted: September 11, 2019; Published: October 2, 2019

\begin{abstract}
African swine fever broke out in China for the first time. China is the world's largest pig breeding country and pork consuming country. Many farmers have insufficient professional knowledge. The traditional feeding mode has unclear understanding of the disease. The measures taken improperly speed up the epidemic. The outbreak has not only caused huge economic losses, but also caused widespread social effects. Many consumers talk about pig color change because they don't understand it, causing waste of social resources. Pig prices are bound to rise and fall, and high-priced pork will directly affect Residents' consumption and food safety are related to every family. It is extremely important to control and eliminate the epidemic as soon as possible. At the same time, we must also recognize the essence and do all kinds of prevention work, especially in the post-epidemic era, and learn from the experience of shrimp. Strive to control the epidemic as soon as possible.
\end{abstract}

Keywords: African Swine Fever, Prevention, Biosafety, Breeding Model, Thinking

\section{Summary of the Incidence of Swine Fever in CHINA}

In August 2018, the first epidemic of African swine fever occurred in China. The epidemic was fierce, with rapid transmission and high mortality, which brought unprecedented pressure on pig breeding and disease prevention and control. The epidemic was developed from the north to the south and exploded. It caused huge economic losses. In just half a year, African swine fever occurred in most parts of China. In this regard, the animal husbandry department has held several emergency meetings to discuss countermeasures. How to prevent the spread of the epidemic and completely eliminate the epidemic is something that animal husbandry and veterinary workers should consider. The current epidemic situation has been stabilized, and at the same time, the ideological sensation cannot be relaxed, and African piglets are eliminated. The epidemic needs to be mobilized together with the whole society. It is necessary to form a cognition, plan, and disposal, and strictly control all barriers, and strive to eliminate the epidemic as soon as possible [1].

\subsection{The African Swine Fever}

African Swine fever (ASF) is an acute, hemorrhagic, and severe infectious disease caused by African swine fever virus infection of domestic pigs and various wild boars (African wild boar, European wild boar, etc.). The World Organisation for Animal Health (OIE) lists it as a statutory report on animal diseases, which is also a major animal epidemic in China. It is characterized by short onset, the most acute and acute infection mortality rate is as high as $100 \%$, clinical manifestations of fever (up to $40-42^{\circ} \mathrm{C}$ ), rapid heartbeat, difficulty breathing, partial cough, serous or mucous purulent eyes and nose Secretions, skin blemishes, lymph nodes, kidneys, and gastrointestinal mucosa are obviously bleeding. The clinical symptoms of African swine fever are similar to those of swine fever, and can only be confirmed by laboratory monitoring. On August 3, 2018, China confirmed the first case of African swine fever, and African swine fever was only infected with pigs [2].

\subsection{The Incidence of Swine Fever in China}

As of July 3, 2019, there were 143 cases of African swine fever in the country, and 1.16 million pigs were culled. At 
present, the epidemic situation in 25 provinces (autonomous regions and municipalities) has been completely blocked. There are only a few places in the country. Hong Kong, Macao, and Taiwan have not reported the African swine fever epidemic.

\section{Current Difficulties in the Prevention and Control of African Swine Fever}

The difficulty in the prevention and control of African swine fever is that it is the first time in China, there is no effective vaccine for prevention and drug control; the second is rapid transmission and wide transmission; the third is rapid onset and the mortality rate is high; Households still hold a certain percentage, which puts pressure on disease regulation and control.

\subsection{Wide Spread}

The transmission of African swine fever is mainly through contact sexual transmission or through air circulation. It can also be transmitted by birds, mosquitoes, etc., and the mode of transmission is mostly the root of the current prevention and control of African swine fever. For the first pigs infected with African swine fever, there is no antibody in the body, and as long as the disease is sick, the mortality rate is high. China is also a large meat-consuming country dominated by pork. Pork accounts for more than 55\% of the total. People who are exposed to unsterilized pathogens during feeding, processing and cooking may spread the disease.

\subsection{Rapid Onset}

The incubation period of African swine fever is 4-19 days. The symptoms are acute, subacute and chronic. The mortality rate is 4-9 days after acute infection, and it suddenly dies. The mortality rate is close to $100 \%$. It is just beginning to be acute in one area. Later, it gradually turned chronic as time went by.

\subsection{Virus Survivability and Virulence}

The virus survives for 2 hours at $56^{\circ} \mathrm{C}, 6$ months at $4{ }^{\circ} \mathrm{C}$, can survive for 2 years at dry room temperature, and can survive almost indefinitely at minus $20^{\circ} \mathrm{C}$. So, say such a stubborn virus, plus The mode of transmission, the speed of onset, and the low bio-safety awareness of people accelerate the speed of disease transmission. Some people use rabbits to do experiments. After 26 generations of transmission, pigs will still die of disease. In some countries, attenuated vaccines are used for vaccination., leading to the strengthening of the virus.

\subsection{The International Disease Situation Is Grim}

Throughout the world, the pressure on prevention and control of African swine fever is also very large. It is understood that 37 countries and regions around the world have reported the African swine fever epidemic to the World Organization for Animal Health. Our neighboring country Russia was asf in 2007, after 10 years. The epidemic continues to occur, causing huge economic losses. In 2017, the Irkutsk region of the Far East discovered asf, which is only 1,000 kilometers [3] from Manzhouli, China.

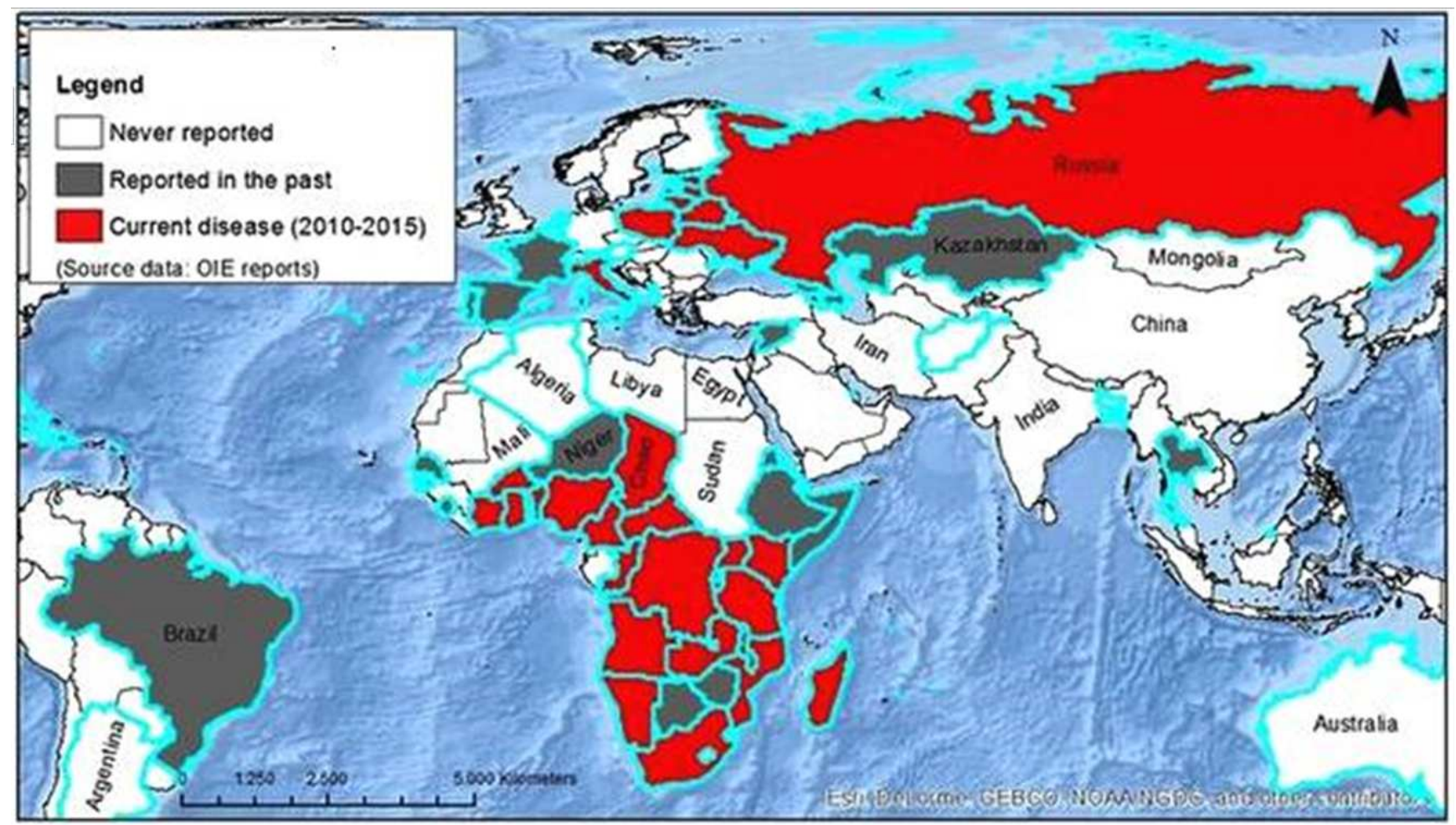

Figure 1. Schematic diagram of global African swine fever in 2010-2015. 
Since April 2019, there have been many African swine fever epidemics in South Africa, Latvia, Belgium, Poland, Cambodia, Ukraine, Hungary, Romania and other countries.

\subsection{China's Breeding Environment and Model}

China's large-scale pig raising accounts for only about $60 \%$, and retailing still occupies a certain proportion. Relatively large-scale farmers, the low biosecurity of the free-range households increases the risk of spread and outbreak of disease, and many retail investors have unclear understanding of the disease. Improper disposal has increased the difficulty of disease control. Moreover, many sows, nursery and fattening pigs are raised together. Once an outbreak occurs, it is difficult to achieve full access, let alone thorough cleaning and disinfection, and purification.

\subsection{The Epidemic Prevention System Is Not Thorough}

The farm should have a complete set of strict epidemic prevention system. However, at present, some pig farm epidemic prevention systems are weak. People and articles directly enter the pig house without any disinfection and isolation measures, which increases the probability of pig contact with the disease. This is also the speed of the disease. A big reason for the quick.

\subsection{Water Feeding}

According to the survey, drowning is a way of spreading swine fever in Africa. Among the epidemic cases reported by the current epidemic, many cases are caused by feeding drowning water. The reason is that there is asf virus in the drowning water, which causes the disease after eating. In September 2018, Shanghai Jinshan and Tan Mou Xue, in the case of receiving the "Notice of Prevention and Control of African Piglets in Jinshan District" issued by the competent authorities twice, still violated the state regulations and their commitments. The kitchen waste (drinking water) imported from other provinces and cities has been used for feeding pigs without high temperature sterilization. On November 16, 2018, the pigs in the farm died of illness and were diagnosed as African swine fever virus infection by the Chinese Center for Health and Epidemiology. The next day, the Information Office of the Ministry of Agriculture and Rural Affairs released the epidemic situation, and Jinshan District initiated the first-level response to the second-level epidemic, and adopted measures such as culling pigs in the epidemic area to control the epidemic. According to the epidemiological investigation of the relevant departments, there is a causal relationship between the occurrence of the African swine fever epidemic and Tan Mou Xue feeding the drowning water (source: Shanghai Jinshan District People's Procuratorate). Some foreign experts analyzed 219 African swine fever cases identified in 2008-2012, and found that $45.6 \%$ of the epidemic was caused by feeding kitchen waste. After the occurrence of the African swine fever epidemic in China, experts conducted a preliminary analysis of the causes of the epidemic. Studies have shown that $62 \%$ of the first 21
African swine fever epidemics in China are related to feeding kitchen waste [4].

\subsection{Human Activities and Consumption Habits}

African swine fever is not infected with humans. It can kill the virus at 70 degrees and above for 20 minutes. Therefore, we only need to cook it after eating. There is no problem, but people invisibly spread it to African piglets indirectly, such as contact with infected pigs. Pork or pork products, which are not properly cleaned and disinfected and then enter the healthy pig house for feeding and feeding pigs, are likely to cause contact infections in pigs, which may lead to disease. According to reports, a number of fast food brands detected the positive detection of African swine fever virus nucleic acid, indicating that the African swine fever virus has penetrated a wide range, but consumers should not be overly panic, as long as the correct disposal, such as high temperature cooking, thoroughly before entering the pig house Disinfection, etc., regular cleaning and disinfection of the pig house environment can effectively prevent infection [5].

\section{Prevention and Control Precautions}

African swine fever is very strong, but as long as we fully understand, reasonably prevent, actively dispose of, and consider multiple times, especially in the post-epidemic period, it is particularly important to strive to eliminate the epidemic as soon as possible.

\subsection{Strict Anti-Epidemic Measures in Farms}

In the old saying, there is a "long-term family wealth, not the gross" does fully reflect the difficulties of the breeding industry, the breeding industry is mainly disease risk, but it is better to treat diseases than disease prevention, disease prevention is important in epidemic prevention. It can be seen that the epidemic prevention system is more important for live animal breeding. The epidemic prevention system and the epidemic prevention system are not on the lips. They are written on the wall and need to be implemented in practice so that each employee has knowledge, conscious and executive. Only in this way can a disease prevention system of a farm be done well, with little or no disease [6].

\subsection{Gradually Eliminate the Free-Range Households and Add Large-Scale Farms}

Large-scale pig farms attach importance to the construction of epidemic prevention systems. For example, feeds are treated with high-temperature treated pellets, feed trucks are discharged from the field, and mechanical feeding is carried out throughout the process. Personnel entering the production area should be thoroughly isolated, bathed, and changed. After thorough disinfection, it can be said that many routes of transmission have been cut off to ensure the safety of the farm. Large-scale breeding farms, pig breeding and fattening are carried out by different subjects. Each subject 
focuses on a single link, constantly refining management and improving efficiency. It can achieve full access, thorough cleaning and disinfection, and disease isolation. The number of free-range households is gradually reduced. This is in line with social development. Throughout the developed countries, scale farming has reached more than $90 \%$, and our country's large-scale farming will also increase year by year [7].

\subsection{Strict Disinfection Before and After Unloading}

The sale of pigs is strictly in accordance with Article 44 of the Animal Epidemic Prevention Law: the vehicles should be cleaned and disinfected in time before loading and after unloading. Practice has shown that $10 \%$ of benzene and phenol, detergents, hypochlorous acid, alkalis and glutaraldehyde are sensitive to African swine fever virus and can effectively kill the virus. Seriously implement the "Transportation of Live Pigs Road Management" for transit disinfection and disposal. Therefore, it is important to perform vehicle disinfection carefully [8].

\subsection{Reduce Pig Transport}

The transportation risk of pigs is very high, especially for sick transportation. If some pigs are in an incubation period, it is difficult for quarantine personnel to judge by looking at the problem. If the transportation is inter-provincial, one or two days, a long four or five days, along the road. Spreading diseases and expanding the area of infection are not conducive to disease control. According to the situation, it is necessary to gradually prohibit the transportation of pigs across provinces and regions, reduce and control the risk of disease transmission, increase the transportation of meat refrigerated and frozen vehicles, and encourage the consumption of chilled products and frozen products.

\subsection{Suspected Symptoms, Correct Disposal}

The transfer of live pigs shall be declared in accordance with the quarantine regulations. The suspected epidemic situation shall be promptly diagnosed, and measures shall be taken in time according to the regulations after diagnosis. Dispose of in strict accordance with the "African Swine Fever Emergency Implementation Plan (2019 Edition)". The General Office of the Ministry of Agriculture and Rural Affairs, the General Office of the Ministry of Finance, "Notice on Doing a Good Job in the Implementation of the Financial Subsidy Policy for the Prevention and Control of African Swine Fever", the "Notice" funds are used for the following three important purposes: quarantine links for pig production areas, and strict implementation of Africa In the clinical examination, investigation and other work of piglets, the pigs in the pigs will be tested for pigs in accordance with the requirements of the epidemic prevention work. In the slaughter and quarantine of pigs, it is necessary to strengthen the on-site inspection of African swine fever. Before the slaughter, the health status of the pigs will be examined in detail.
During the slaughter process, the clinical symptoms and typical pathological changes of suspected African swine fever will be examined, and the pork products or blood of the quarantine link will be sampled. In the supervision of transportation vehicles, it is necessary to strengthen the designated passages, the construction of inter-provincial temporary animal health supervision and inspection stations at the highway animal health supervision and inspection station, enrich the work force of the inter-provincial animal health supervision station, improve working conditions, and strictly implement the 24-hour duty system. Strengthen the supervision of pig transport vehicles, increase the inspection and cleaning and disinfection of pigs and their vehicles across the province, and effectively reduce the risk of cross-regional spread of the epidemic [9].

\subsection{Strict and Harmless Disposal of Sick and Dead Livestock}

Harmless treatment refers to the treatment of dead animals and related animal products by physical and chemical methods, eliminating the pathogens carried by them, and eliminating the harm of animal carcasses. There are many advantages in the harmless treatment. The main reason is to control diseases and protect the environment. To improve food safety and waste recycling, in a highly developed social system, harmlessness is an operating point that fully reflects the way people live in harmony with nature and respects and respects nature [10].

\subsubsection{Controlling the Source of Infection}

The spread of disease is mainly the source of infection, the route of transmission and susceptible animals. The sick and dead animals are the main source of infection. If it can be disposed of harmlessly according to regulations, and avoid new sources of infection, it is very beneficial for disease control.

\subsubsection{Reduce Environmental Pollution}

If the dead animals are littered everywhere, the bodies will be spoiled and fermented, polluting rivers, land and air, mosquitoes will multiply in the summer, stinking, and increasing environmental pressure. Now the country is proposing a rural revitalization strategy, and we must build our country into rural beauty and agriculture. Strong and peasant-rich new villages are essential for the harmless disposal of sick and dead animals [11].

\subsection{Popularize Knowledge and Let Consumers Know}

There are many factors in the outbreak of the disease, such as unclear understanding, poor implementation, and lax implementation. First of all, there should be an understanding of this situation, what it is, how it spreads, those hazards, etc., and it must rise to a universal popularity. In this case, this work is very difficult, but it must be done, and only the whole society can work together to eradicate the African swine fever epidemic [12]. 


\subsection{Ways and Means of Foreign Countries to Eliminate African Swine Fever}

For African swine fever, since it was first reported in 1921, it has swept most countries and regions on the earth. At present, there is no effective control of vaccines to prevent this disease, and there is no obvious drug treatment. All of them rely on culling and harmless treatment. Of the 68 countries and territories where the outbreak has occurred, only 13 countries have achieved eradication. Some countries have killed all the pigs in the country in order to eliminate it, such as the African swine fever epidemic in Malta in 1978, and the pigs raised in the country in 1979. All culled, creating a precedent for a country to cull a livestock to eliminate an infectious disease. Brazil (1978-1985) spent seven years eradicating African swine fever, Spain (1985-1995) spent 10 years to eliminate African swine fever, and Russia reported the African swine fever epidemic for the first time since 2007, and the epidemic still does not get control. Therefore, it is very difficult to eliminate African swine fever in this situation in a short period of time. The following six aspects of the elimination of African swine fever in Spain are worthy of reference: 1 Improve the veterinary clinical team 2 Accurately test all farms 3 Improve the health level of the farm (reorganize) 4 Completely eliminate the source of infection 5 Strictly control the movement of pigs 6 pairs of Africa The piglet is promoted in science [13].

\section{African Pigs Think Deeply}

In the post-epidemic period, it is necessary to take into account the frequent occurrence and frequent occurrence of the epidemic, which will bring a painful blow to the pig breeding industry. The amount of pigs raised will be drastically reduced, the price of pork will rise sharply, and the capital will be swarmed in. Only by standardizing cultivation, practicing internal strength, and making progress, can we to make the breeding industry bigger and stronger, it can promote the healthy development of pig breeding and the stability of social consumption.

\subsection{Pork Price Rises}

It is expected that pork prices will rise sharply this year, and it is very likely to break through the historical record. China is the world's largest pork consumer. With the outbreak and spread of African swine fever, sow stocks and pig breeding will be drastically reduced. Declining to the lowest value in a decade will also strictly control the importing countries, and the relationship of less than demand is inevitable.

The Ministry of Agriculture and Rural Agriculture has issued seven measures to stabilize pig production, also to prevent the rise in pig prices and cause certain social problems.

Considering that the epidemic has a certain impact on pig breeding, such as the lower survival rate, the pig breeding rate is reduced, try to avoid the fact that although the price of the pig is high, but the farmers cannot make money, the disease is wasting social resources, and reduced The output situation creates a vicious circle.

\subsection{Social and Price Stability}

Further increase the storage and storage of pork. In March 2019 , it started three times of pork storage and storage. Due to the reduction of domestic pork output, the country will inevitably increase imports, and the 16 countries of the United States, Germany, Brazil and other countries will be allowed to export pork products, 2019 In March, imports of 23,846 tons of US pork, a $90 \%$ increase over the same period, the largest order in history. On March 22, 24.99 tons of the first batch of pork products from Portugal landed in Tianjin. Recently, we are concerned about the new US agricultural products procurement in Chinese enterprises. Contains pork [14].

\subsection{Enlightenment from the Early Death Syndrome of Prawn to African Swine Fever}

There is a very profound example in front of us. In 2008, it was a watershed for China's shrimp farming industry. The arrival of ems (early death syndrome) directly sentenced the death penalty of Chinese shrimp farming industry, and the breeding death rate was $70 \%$. As long as the survival rate is higher than $30 \%$, you can make money. Since then, the people have bid farewell to cheap prawns, and the price of prawns has turned over several times. Ten years have passed, the disease has still not been controlled, the price of shrimp has risen from the original 8-10 yuan / $\mathrm{kg}$ to $80-100$ yuan / kg, so that ordinary people are discouraged, and 50 grams / more shrimp 200 yuan/jin. With the outbreak of African swine fever, our pigs will be like the prawn, the price will fly directly, it should be worth pondering and considering.

\subsection{Finding a Suitable Alternative}

While stabilizing the production of pigs, it is necessary to guarantee the supply of meat such as poultry and beef and mutton. In particular, poultry meat, because of its high protein content, low in fat, cholesterol, and energy, belongs to "white meat," which is inexpensive and should be promoted. Looking back at the proportion of meat consumption in China, it is enough to see the reduction of pork and the increase of poultry meat. The poultry meat is mainly white feather broiler. The ratio of meat to meat is $1.6: 1$, which is the most economical feed among all meats. It is expected that the consumption and proportion of white feather broilers should continue to increase in the future [15]. 


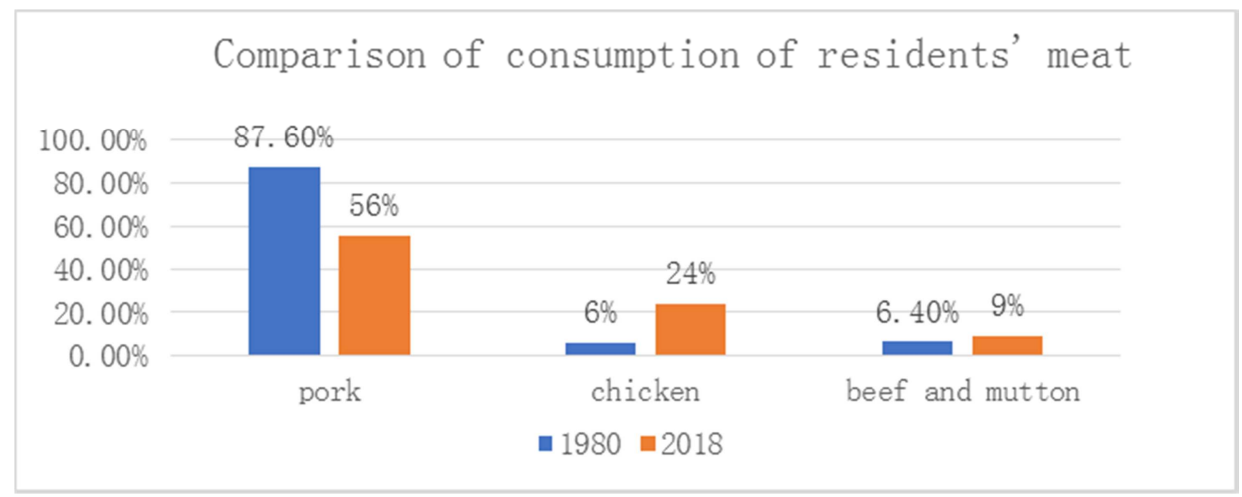

Figure 2. Residents' consumption of meat.

\section{Summary}

Although the African pig swine epidemic is in a raging situation, as long as the veterinarians fully understand, do a good job of disinfection, eliminate the source of infection; grasp the epidemic prevention system, cut off the route of transmission; do a good job of feeding and management, and increase the ability of pigs to resist disease. urther increase the proportion of large-scale farmers, use the all-in and all-out feeding mode, promote the mechanization process in an orderly and reasonable manner, ensure sufficient empty house period, increase the control of African piglets, and all the people know, treat them correctly, and the government rationally and scientifically guides Keep a close eye on international developments, do a good job in vaccine research, and strive to eliminate the African swine fever epidemic as soon as possible.

\section{References}

[1] Yi Benchi, Zhang Ting, Liu Tao, Wang Rui. Diagnosis and treatment of swine disease and rational use of drugs [m]. Henan: Henan Science and Technology Press. 2013: 85-90.

[2] Zhang Xiujuan. Knowledge of prevention and control of African swine fever [m]. Beijing: China Agricultural Science and Technology Press. 2019: 5-12.

[3] Ge Shengqiang, Li Jinming, Ren Yujie et al. The prevalence and research status of African swine fever in Russia [j]. Bulletin of Microbiology, 2017, 44 (12): 3067-3076

[4] EU: Personnel is an important factor affecting the spread of African swine fever [j]. Northern Animal Husbandry, 2019, 07: 18

[5] Fu Xinchun, talk about the prevention and control measures of African swine fever [j]. Modern Animal Husbandry Science and Technology, 2019, 08: 134-136.

[6] Yu Pengtao, Duan Qingbin, African piglet biosafety prevention and control measures [j]. Today animal husbandry and veterinary, 2019, 07: 46-47.
[7] He Taobo, on how to prevent and control African swine fever [j]. Livestock and poultry industry, 2019, 07: 97.

[8] Sun Pengcheng, Analysis of the diagnosis and prevention measures of African swine fever [j]. Chinese animal health, 2019, 07: 27-28.

[9] Cao Yixiang, diagnosis and prevention of African swine fever [j]. Chinese animal health, 2018, 12: 24-25.

[10] Chen Yuanyuan, analysis of grassroots prevention and control measures in African swine fever [j]. China Animal Health, 2018, 12: 17-18.

[11] Wang Jinlong, prevention and control measures for African swine fever [j]. Chinese animal health, 2018, 12: 28-29.

[12] The General Office of the State Council issued the "Opinions on Strengthening the Prevention and Control of African Pigs" [j]. Northern Animal Husbandry, 2019, 07: 06.

[13] Cheng Jianguo, the impact and challenges of African swine fever on China's pig industry and countermeasures [j]. Scientific breeding, 2019, 07: 5-7.

[14] Wang Junxi, Wang Zhiliang. African swine fever [m]. Beijing: China Agriculture Press. 2014: 98-101.

[15] Ministry of Agriculture and Rural Affairs: The annual supply of pork is relatively tight and leads to increase the production of poultry substitutes [j]. Today's pig industry, 2019, 07: 6 .

\section{Biography}

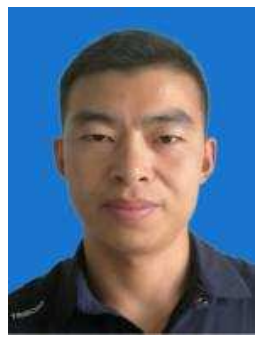

Shi Haidong: Date of birth: December 1985, gender: male, education: undergraduate; Birthplace: Haimen, Jiangsu; Title: veterinarian, research direction: animal husbandry and veterinary. he author has studied animal husbandry and veterinary for many years, and has often gone deep into the front line, publishing more than 70 professional papers and publishing 2 monographs. 